\title{
Case Reviews in Ophthalmology: Expert Consult - Online and Print, 1e (Expert Consult Title: Online + Print) Editors: Neil J. Friedman and Peter K. Kaiser, 2009, ISBN-10: 1437726135, Saunders Elsevier
}

\author{
Asaf Achiron • Danny Goldhaber
}

Received: 5 December 2014 / Accepted: 11 December 2014 / Published online: 31 December 2014

(C) Springer-Verlag Berlin Heidelberg 2014

https://www.inkling.com/store/book/case-reviews-inophthalmology-friedman-kaiser-1st/? chapter $\mathrm{Id}=$ cb9e311c5e7345fda11daeb9c660be47

"Case Reviews in Ophthalmology: Expert Consult", by Friedman and Kaiser. Reviewed in its online version.

Contents The book consists of 116 cases that provide a comprehensive review of six main topics: optics/refraction, neuroophthalmology/orbit, pediatrics/strabismus, external disease/ adnexa, anterior segment, and posterior segment. The cases demonstrate common and vision-threatening ocular conditions that the certified ophthalmologist must be able to recognize and treat.

Format The format reviewed is that of an interactive eBook that can be read anywhere (computer, iOS, Android) and without internet, thanks to its offline access format. The reader can search, navigate, take notes, and highlight important text in the eBook.

Aim of the book To help fill in some knowledge gaps for doctors preparing for the oral board review.

A. Achiron · D. Goldhaber

Sackler School of Medicine, Tel-Aviv University, Tel-Aviv, Israel

A. Achiron $(\bowtie)$

Department of Ophthalmology, the Edith Wolfson Medical Center,

Holon, Israel

e-mail: achironasaf@gmail.com
Reviews and thoughts This eBook offers interactive reading in the form of quizzes. Every case consists of a patient description followed by self-test questions. The questions focus mainly on correctly diagnosing and managing patients. The cases also test for other possible diagnoses, specific clinical signs, and prognoses. After offering an oral response to a question, the reader clicks the answer button to reveal the book's answer, which is clinically oriented and in the same format as the real exams.

For residents, the cases are a useful way to practice important subjects and stay focused (or awake) after a long work day. It also provides for a good use of one's commute time. We agree with the author's belief that the case-based method is a valuable technique for learning and reinforcing core concepts. The cases and questions are written in a stepwise fashion, and the reader can practice the cases orally, thus simulating a real testing experience. The fact that these cases were written by doctors who are both experts in their fields and examiners on oral board reviews is a great advantage.

\section{Conclusions}

Though this is not a comprehensive textbook and only covers the most general and important subjects, it is excellent for studying and practicing in a case-based format. The book's answers give important clinical tips, which even experienced physicians can benefit from. 